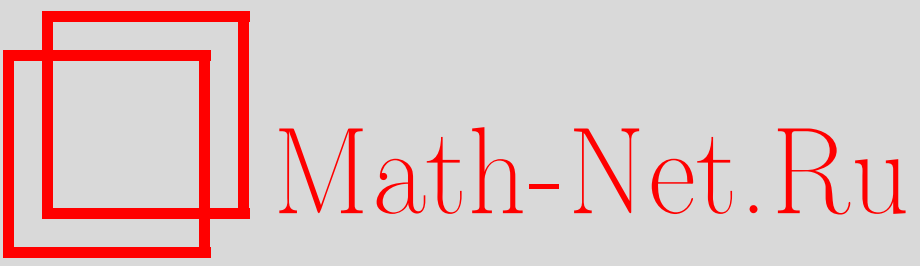

O. E. Barndorff-Nielsen, J. Pedersen, Meta-times and extended subordination, Teoрия вероятн. и ее примен., 2011, том 56, выпуск 2, 398-407

DOI: https://doi.org/10.4213/tvp4384

Использование Общероссийского математического портала Math-Net.Ru подразумевает, что вы прочитали и согласны с пользовательским соглашением

http://www.mathnet.ru/rus/agreement

Параметры загрузки:

IP : 34.227 .88 .159

26 апреля 2023 г., 16:43:41

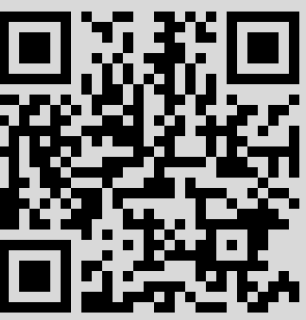




\section{СПИСОК ЛИТЕРАТУРЫ}

1. Graversen S. E., Peskir G., Shiryaev A. N. Stopping Brownian motion without anticipation as close as possible to its ultimate maximum. - Теория вероятн. и ее примен., 2000 , т. 45 , в. 1 , с. $125-136$.

2. Pedersen J. L. Optimal prediction of the ultimate maximum of Brownian motion. Stochastics and Stochastics Rep., 2003, v. 75, № 4, p. 205-219.

3. Shiryaev A.N. Quickest detection problems in the technical analysis of the financial data. - Mathematical Finance-Bachelier Congress (Paris, 2000). Berlin: SpringerVerlag, 2002, p. 487-521.

4. Урусов М.А. Об одном свойстве момента достижения максимума броуновским движением и некоторых задачах оптимальной остановки. - Теория вероятн. и ее примен., 2004, т. 49, в. 1, с. 184-190.

5. Ширяев A.Н. Об условно-экстремальных задачах скорейшего обнаружения непредсказуемых моментов у наблюдаемого броуновского движения. - Теория вероятн. и ее примен., 2008, т. 53 , в. 4 , с. $751-768$.

6. Ширяев А.Н. Статистический последовательный анализ. М.: Наука, 1976, 272 с.

7. du Toit J., Peskir G. The trap of complacency in predicting the maximum. - Ann. Probab., 2007, v. 35, № 1, p. 340-365.

8. du Toit J., Peskir G. Predicting the time of the ultimate maximum for Brownian motion with drift. - Mathematical Control Theory and Finance. Berlin: SpringerVerlag, 2008, p. 95-112.

9. Ширяев А.Н. Основы стохастической финансовой математики. М.: Фазис, 1998, $1008 \mathrm{c}$.

10. Peskir G. On reflecting Brownian motion with drift. - Proceedings of the 37th ISCIE International Symposium on Stochastic Systems Theory and its Applications. Kyoto: ISCIE, 2005 , p. $1-5$.

11. Graversen S. E., Shiryaev A.N. An extension of P. Lévy's distributional properties to the case of a Brownian motion with drift. - Bernoulli, 2000, v. 6, № 4, p. 615-620.

12. Peskir G., Shiryaev A.N. Optimal Stopping and Free-Boundry Problems. Basel: Birkhäuser, 2006, 500 p.

13. Дьъкин Е. Б. Марковские процессы. М.: Физматгиз, 1963, 859 с.

Поступила в редакцию

9.XII.2009

Исправленный вариант 6.X.2010

(c) 2011 г.

BARNDORFF-NIELSEN O. E. , PEDERSEN J.* META-TIMES AND EXTENDED SUBORDINATION

Обсуждается задача определения субординатора однородного базиса Леви с помощью неотрицательного однородного базиса Леви. Приводится явная конструкция, обобщающая обычный одномерный случай. Эта конструкция задействует некоторые случайные замены метавремени.

Ключевые слова и фразы: базисы Леви, листы Леви, замена метавремени.

* Department of Mathematical Sciences, University of Aarhus, Denmark; e-mail: oebn@imf.au.dk, jan@imf.au.dk 
1. Introduction. In recent years the fundamental concept of subordination of a Lévy process by a subordinator has been generalized in many directions; see, e.g., [3], [4], [8]. Related to this, Barndorff-Nielsen [2] considered the following problem: Given an $\mathbf{R}^{d}$ valued homogeneous Lévy basis $\Lambda_{X}=\left\{\Lambda_{X}(A): A \in \mathscr{B} \mathrm{b}\left(\mathbf{R}^{k}\right)\right\}$, and an independent $\mathbf{R}_{+}$valued homogeneous Lévy basis $\Lambda_{T}=\left\{\Lambda_{T}(A): A \in \mathscr{B}_{\mathrm{b}}\left(\mathbf{R}^{k}\right)\right\}$ how should one define subordination of $\Lambda_{X}$ by $\Lambda_{T}$ ?

Let us first consider the case $k=1$. There are Lévy processes $X=\left\{X_{t}: t \geqslant 0\right\}$ and $T=\left\{T_{t}: t \geqslant 0\right\}$ associated with $\Lambda_{X}$ and $\Lambda_{T}$ in the sense that

$$
X_{t}=\Lambda_{X}((0, t]) \quad \text { and } \quad T_{t}=\Lambda_{T}((0, t])
$$

for $t \geqslant 0$. Thus, we can simply define a subordinated process $Y=\left\{Y_{t}: t \geqslant 0\right\}$ in the usual way as $Y_{t}=X_{T_{t}}$. However, when $k \geqslant 2$ there is no immediate analogue. To see this, note that there are so-called Lévy sheets $X=\left\{X_{t}: t \in \mathbf{R}_{+}^{k}\right\}$ and $T=\left\{T_{t}: t \in \mathbf{R}_{+}^{k}\right\}$ associated with $\Lambda_{X}$ and $\Lambda_{T}$, and these are defined as in (1.1), where $(0, t]$ now is an interval in $\mathbf{R}^{k}$. But $T_{t}$ is one-dimensional while $t$ is $k$-dimensional, thus excluding the possibility of defining $Y_{t}$ as $X_{T_{t}}$ when $k \geqslant 2$. Barndorff-Nielsen argued that one should not construct a subordinated process; rather, the appropriate concept is a subordinated random measure $M=\left\{M(A): A \in \mathscr{B}_{\mathrm{b}}\left(\mathbf{R}^{k}\right)\right\}$ defined in such a way that conditional on $\Lambda_{T}, M\left(A_{1}\right), \ldots, M\left(A_{n}\right)$ are independent for all disjoint $A_{1}, \ldots, A_{n}$, and the distribution of $M(A)$ for $A \in \mathscr{B}_{b}\left(\mathbf{R}_{+}^{k}\right)$ is $\mu^{\Lambda_{T}(A)}$, where $\mu=\mathscr{L}\left(\Lambda_{X}((0, e])\right)$ and $e=(1, \ldots, 1) \in \mathbf{R}_{+}^{k}$ is the vector of ones.

In the present paper we give an explicit construction in terms of $\Lambda_{X}$ and $\Lambda_{T}$ of Barndorff-Nielsen's subordinated measure $M$. For notational convenience, instead of considering $M$ and $\Lambda_{X}$ as Lévy bases on $\mathbf{R}^{k}$ we look at the restriction to $\mathbf{R}_{+}^{k}$; the general case follows trivially from this. Specifically, we argue that a natural definition of $M=\left\{M(A): A \in \mathscr{B}_{b}\left(\mathbf{R}_{+}^{k}\right)\right\}$ is $M(A)=\Lambda_{X}\left(\phi_{T}^{-1}(A)\right)$, where $\phi_{T}: \mathbf{R}_{+}^{k} \rightarrow \mathbf{R}^{k}$ is a (random) mapping satisfying that $\operatorname{Leb}\left(\phi_{T}^{-1}(A)\right)=\Lambda_{T}(A)$ for $A \in \mathscr{B}_{\mathrm{b}}\left(\mathbf{R}_{+}^{k}\right)$. We also use the notation $\mathbf{T}=\phi_{T}^{-1}$ for the inverse image of $\phi_{T}$. In a sense one can think of $\mathbf{T}$ as a kind of meta-time change, replacing time changes in the case $k=1$. We show that this definition generalizes the case $k=1$ in a natural way and, in particular, that $M$ is a homogeneous Lévy basis.

This construction gives emphasis to the viewpoint that in the multiparameter case $k \geqslant 2$ the right concept is a subordinated measure instead of subordinated process.

In Section 2 we recall the definitions of homogeneous Lévy sheets and bases and show that these are in one-to-one correspondence. To pave the way for the analysis of $M$ we state in Section 3 a lemma showing that it is possible to define a mapping $\phi_{T}$ with the above properties. Section 4 is about meta-time changes, meaning that we consider the measure $M$ for a fixed outcome of $T$. Although meta-times exist for any $T$ they are in full generality somewhat involved to define explicitly. However, in practice the most important case is when the measure induced by $T$ is the sum of a constant times Lebesgue measure and a discrete measure. In this case alternative useful representations of $M$ are given. In the last section it is shown that $M$ is a homogeneous Lévy basis.

2. Homogeneous Lévy sheets and Lévy bases. Let $d$ and $k$ be positive integers. For $x=\left(x^{1}, \ldots, x^{d}\right)$ and $y=\left(y^{1}, \ldots, y^{d}\right)$ in $\mathbf{R}^{d}$ let $\langle x, y\rangle$ denote their inner product and $|x|$ the corresponding norm. Let $D=\left\{x \in \mathbf{R}^{d}:|x| \leqslant 1\right\}$. Throughout the paper all random variables are defined on a common probability space $(\Omega, \mathscr{F}, \mathbf{P})$. Let $\mathscr{L}(X)$ denote the law of a random vector $X$. For a set $S$ and two families $\left\{X_{t}: t \in S\right\}$ and $\left\{Y_{t}: t \in S\right\}$ of random vectors with $X_{t}$ and $Y_{t}$ in $\mathbf{R}^{d}$ write $\left\{X_{t}: t \in S\right\} \stackrel{\mathscr{D}}{=}\left\{Y_{t}: t \in S\right\}$ if the finite dimensional marginals are the same. We say that $\left\{X_{t}: t \in S\right\}$ is a modification of $\left\{Y_{t}: t \in S\right\}$ if $X_{t}=Y_{t}$ a.s. for all $t \in S$. Let $\widehat{\mu}$ denote the characteristic function of a distribution $\mu$ on $\mathbf{R}^{d}, \widehat{\mu}(z)=\int_{\mathbf{R}^{d}} e^{\mathrm{i}\langle z, x\rangle} \mu(\mathrm{d} x)$ for $z \in \mathbf{R}^{d}$.

Let $\operatorname{ID}\left(\mathbf{R}^{d}\right)$ denote the class of $d$-dimensional infinitely divisible distributions. Recall that a distribution $\mu$ on $\mathbf{R}^{d}$ is in $\operatorname{ID}\left(\mathbf{R}^{d}\right)$ if and only if $\widehat{\mu}$ is given by $\widehat{\mu}(z)=\exp \left[-z \Sigma z^{\top} / 2+\right.$ 
$\left.\mathrm{i}\langle\gamma, z\rangle+\int_{\mathbf{R}^{d}} g(z, x) \nu(\mathrm{d} x)\right], z \in \mathbf{R}^{d}$, where $g(z, x)=e^{\mathrm{i}\langle z, x\rangle}-1-\mathrm{i}\langle z, x\rangle 1_{D}(x),{ }^{\top}$ denotes the transpose, and $(\Sigma, \nu, \gamma)$ is the characteristic triplet of $\mu$; that is, $\Sigma$ is a $d \times d$ nonnegative definite matrix, $\nu$ is a Lévy measure on $\mathbf{R}^{d}$ and $\gamma \in \mathbf{R}^{d}$. Denote the entries of $\Sigma$ by $\Sigma^{i j}$ and the coordinates of $\gamma$ by $\gamma^{j}$ for $i, j=1, \ldots, d$. For $t \geqslant 0$ and $\mu \in \operatorname{ID}\left(\mathbf{R}^{d}\right), \mu^{t}$ denotes the distribution in $\operatorname{ID}\left(\mathbf{R}^{d}\right)$ with $\widehat{\mu^{t}}=\widehat{\mu}^{t}$.

For $a=\left(a^{1}, \ldots, a^{k}\right) \in \mathbf{R}_{+}^{k}$ and $b=\left(b^{1}, \ldots, b^{k}\right) \in \mathbf{R}_{+}^{k}$ write $a \leqslant b$ if $a^{j} \leqslant b^{j}$ for all $j$ and $a<b$ if $a^{j}<b^{j}$ for all $j$, and define the half-open interval $\left.] a, b\right]$ as $\left.] a, b\right]=\{t \in$ $\left.\mathbf{R}_{+}^{k}: a<t \leqslant b\right\}$. Let $[a, b]=\left\{t \in \mathbf{R}_{+}^{k}: a \leqslant t \leqslant b\right\}$.

For $F=\left\{F_{t}: t \in \mathbf{R}_{+}^{k}\right\}$ with $F_{t} \in \mathbf{R}^{d}$ and $a \leqslant b$ define the increment of $F$ over $\left.] a, b\right]$, $\Delta_{a}^{b} F$, as

$$
\Delta_{a}^{b} F=\sum_{\epsilon_{1}=0}^{1} \ldots \sum_{\epsilon_{k}=0}^{1}(-1)^{k-\left(\epsilon_{1}+\cdots+\epsilon_{k}\right)} F_{\left(c^{1}\left(\epsilon_{1}\right), \ldots, c^{k}\left(\epsilon_{k}\right)\right)},
$$

where $c^{j}(1)=b^{j}$ and $c^{j}(0)=a^{j}$. For example, if $k=1$, then $\Delta_{a}^{b} F=F_{b}-F_{a}$ and when $k=2$ we have $\Delta_{a}^{b} F=F_{\left(b^{1}, b^{2}\right)}+F_{\left(a^{1}, a^{2}\right)}-F_{\left(a^{1}, b^{2}\right)}-F_{\left(b^{1}, a^{2}\right)}$. Let $\mathscr{A}=\left\{t \in \mathbf{R}_{+}^{k}: t^{j}=0\right.$ for some $j$ \}. For $\mathscr{R}=\left(R_{1}, \ldots, R_{k}\right)$, where $R_{j}$ is either $\leqslant$ or $>$, write $a \mathscr{R} b$ if $a^{j} R_{j} b^{j}$ for all $j$.

We say that $F=\left\{F_{t}: t \in \mathbf{R}_{+}^{k}\right\}$ is lamp if the following three conditions are satisfied: (i) for $t \in \mathbf{R}_{+}^{k}$ the limit $F(t, \mathscr{R})=\lim _{u \rightarrow t, t \mathscr{R} u} F_{u}$ exists for each of the $2^{k}$ relations $\mathscr{R}=\left(R_{1}, \ldots, R_{k}\right)$, where $R_{j}$ is either $\leqslant$ or $>$ (here we let $F(t, \mathscr{R})=F_{t}$ if there is no $u$ with $t \mathscr{R} u$ ); (ii) $F_{t}=F(t, \mathscr{R})$ for $\mathscr{R}=(\leqslant, \ldots, \leqslant)$; (iii) $F_{t}=0$ for $t \in \mathscr{A}$. Here lamp stands for limits along monotone paths. This is the multiparameter analogue of being càdlàg. See [1] for references to the literature on lamp trajectories. When $F$ is lamp and $t \in \mathbf{R}_{+}^{k} \backslash \mathscr{A}$ define $\Delta_{t} F=\lim _{n \rightarrow \infty} \Delta_{t_{n}}^{t} F$, where $t_{n}$ is any sequence with $t_{n} \rightarrow t$ and $t_{n}<t$. If $F$ is continuous at the point $t$, then $\Delta_{t} F=0$ but the converse is not true, that is, we can have $\Delta_{t} F=0$ without $F$ being continuous at $t$.

D e f i n i t i o n 2.1. Let $X=\left\{X_{t}: t \in \mathbf{R}_{+}^{k}\right\}$ be a family of random vectors in $\mathbf{R}^{d}$. We say that $X$ has independent increments if $X_{t}=0$ for all $t \in \mathscr{A}$ a.s. and $\Delta_{a_{1}}^{b_{1}} X, \ldots, \Delta_{a_{n}}^{b_{n}} X$ are independent whenever $n \geqslant 2$ and $\left.\left.\left.] a_{1}, b_{1}\right], \ldots,\right] a_{n}, b_{n}\right]$ are disjoint; if in addition $X$ is continuous in probability and $\Delta_{t+a}^{t+b} X \stackrel{\mathscr{D}}{=} \Delta_{a}^{b} X$ for all $a, b, t \in \mathbf{R}_{+}^{k}$ with $a \leqslant b$, then $X$ is called an $\mathbf{R}^{d}$-valued homogeneous Lévy sheet in law on $\mathbf{R}_{+}^{k}$, and if also almost all sample paths are lamp, then $X$ is called an $\mathbf{R}^{d}$-valued homogeneous Lévy sheet on $\mathbf{R}_{+}^{k}$.

A homogeneous Lévy sheet is a special case of the additive processes considered in $[1$, p. 5], and of the Lévy sheets considered in [5] (in the case $k=2$ ). In fact, a process satisfying all the above conditions except the homogeneity condition $\Delta_{t+a}^{t+b} X \stackrel{\mathscr{D}}{=} \Delta_{a}^{b} X$ would be called a Lévy sheet by Dalang and Walsh [5]. It follows, e.g., from [1, Proposition 4.1] that any homogeneous Lévy sheet in law has a modification which is a homogeneous Lévy sheet. It is easily seen that if $X=\left\{X_{t}: t \in \mathbf{R}_{+}^{k}\right\}$ is a homogeneous Lévy sheet in law, then $X_{t}=\Delta_{0}^{t} X$ a.s. for all $t \in \mathbf{R}_{+}^{k}$; moreover $\mathscr{L}\left(\Delta_{a}^{b} X\right) \in \operatorname{ID}\left(\mathbf{R}^{d}\right)$ for all $a, b \in \mathbf{R}_{+}^{k}$ with $a \leqslant b$ and there is a $\mu \in \operatorname{ID}\left(\mathbf{R}^{d}\right)$ such that $\mathscr{L}\left(\Delta_{a}^{b} X\right)=\mu^{\operatorname{Leb}(] a, b])}$ for all such $a$ and $b$, where Leb denotes Lebesgue measure on $\mathbf{R}^{k}$. We say that $X$ is associated with $\mu$ or with the characteristic triplet of $\mu$.

D e f i n i t i o n 2.2. Let $\Lambda=\left\{\Lambda(A): A \in \mathscr{B}_{b}\left(\mathbf{R}_{+}^{k}\right)\right\}$, where $\mathscr{B}_{\mathrm{b}}\left(\mathbf{R}_{+}^{k}\right)$ is the set of bounded Borel sets in $\mathbf{R}_{+}^{k}$, denote a family of random vectors in $\mathbf{R}^{d}$. We call $\Lambda$ an $\mathbf{R}^{d}$-valued homogeneous Lévy basis on $\mathbf{R}_{+}^{k}$ if the following conditions are satisfied: (i) $\Lambda\left(A_{1}\right), \ldots, \Lambda\left(A_{n}\right)$ are independent whenever $A_{1}, \ldots, A_{n} \in \mathscr{B}_{\mathrm{b}}\left(\mathbf{R}_{+}^{k}\right)$ are disjoint; (ii) $\Lambda\left(\bigcup_{n=1}^{\infty} A_{n}\right)=\sum_{n=1}^{\infty} \Lambda\left(A_{n}\right)$ a.s. whenever $A_{1}, A_{2}, \ldots \in \mathscr{B}_{\mathrm{b}}\left(\mathbf{R}_{+}^{k}\right)$ are disjoint with $\bigcup_{n=1}^{\infty} A_{n} \in \mathscr{B}_{\mathrm{b}}\left(\mathbf{R}_{+}^{k}\right)$ (here the series converges almost surely); (iii) for all $t \in \mathbf{R}_{+}^{k}$ and $A \in \mathscr{B}\left(\mathbf{R}_{+}^{k}\right)$ we have $\Lambda(A) \stackrel{\mathscr{D}}{=} \Lambda(t+A)$.

If $\Lambda$ is a homogeneous Lévy basis, then $\mathscr{L}(\Lambda(A)) \in \operatorname{ID}\left(\mathbf{R}^{d}\right)$ for all $A \in \mathscr{B}_{\mathrm{b}}\left(\mathbf{R}_{+}^{k}\right)$. Moreover, there is a $\mu \in \operatorname{ID}\left(\mathbf{R}^{d}\right)$ such that $\mathscr{L}(\Lambda(A))=\mu^{\operatorname{Leb}(A)}$ for all $A \in \mathscr{B}_{\mathrm{b}}\left(\mathbf{R}_{+}^{k}\right)$. 
We say that the homogeneous Lévy basis is associated with $\mu$ or its characteristic triplet. Finally, recall that [9] calls $\left.\Lambda=\left\{\Lambda(A): A \in \mathscr{B}_{\mathrm{b}}\left(\mathbf{R}_{+}^{k}\right)\right)\right\}$ an independently scattered ID random measure if it satisfies (i) and (ii) of Definition 2.2 and $\mathscr{L}(\Lambda(A)) \in \operatorname{ID}\left(\mathbf{R}^{d}\right)$ for all $A \in \mathscr{B}_{\mathrm{b}}\left(\mathbf{R}_{+}^{k}\right)$. For simplicity we refer to this as a Lévy basis.

The following shows that, not surprisingly, there is a one-to-one correspondence between homogeneous Lévy sheets (in law) and homogeneous Lévy bases.

Theorem 2.1. Let $X=\left\{X_{t}: t \in \mathbf{R}_{+}^{k}\right\}$ be a homogeneous Lévy sheet associated with $\mu \in \operatorname{ID}\left(\mathbf{R}^{d}\right)$ with characteristic triplet $(\Sigma, \nu, \gamma)$. Let

$J(C)=\#\left\{\left(t, \Delta_{t} X\right): t \in \mathbf{R}_{+}^{k} \backslash \mathscr{A},\left(t, \Delta_{t} X\right) \in C\right.$ and $\left.\Delta_{t} X \neq 0\right\} \quad$ for $C \in \mathscr{B}\left(\mathbf{R}_{+}^{k} \times \mathbf{R}^{d}\right)$.

Then we have the following.

1) $J=\left\{J(C): C \in \mathscr{B}\left(\mathbf{R}_{+}^{k} \times \mathbf{R}^{d}\right)\right\}$ is a Poisson random measure with intensity measure Leb $\times \nu$.

$$
\begin{aligned}
& \text { 2) Let } \nu^{1}(B)=\nu(B \cap D) \text { and } \nu^{2}(B)=\nu\left(B \cap D^{c}\right) \text { for } B \in \mathscr{B}\left(\mathbf{R}^{d}\right) \text {. Define } \\
& X_{t}^{1}=\int_{[0, t] \times \mathbf{R}^{d}} y 1_{D}(y)(J-\operatorname{Leb} \times \nu)(\mathrm{d}(s, y)), \quad X_{t}^{2}=\int_{[0, t] \times \mathbf{R}^{d}} y 1_{D^{c}}(y) J(\mathrm{~d}(s, y)) .
\end{aligned}
$$

We then have that $X_{t}=X_{t}^{1}+X_{t}^{2}+X_{t}^{\mathrm{g}}+t \gamma$, where $\left\{X_{t}^{\mathrm{g}}: t \in \mathbf{R}_{+}^{k}\right\},\left\{X_{t}^{1}: t \in \mathbf{R}_{+}^{k}\right\}$ and $\left\{X_{t}^{2}: t \in \mathbf{R}_{+}^{k}\right\}$ are independent, $\left\{X_{t}^{\mathrm{g}}: t \in \mathbf{R}_{+}^{k}\right\}$ is a homogeneous Lévy sheet associated with $(\Sigma, 0,0)$ and $\left\{X_{t}^{i}: t \in \mathbf{R}_{+}^{k}\right\}$ is a homogeneous Lévy sheet associated with $\left(0, \nu^{i}, 0\right)$ for $i=1,2$.

3) There exists one and up to modification only one homogeneous Lévy basis $\Lambda=$ $\left\{\Lambda(A): A \in \mathscr{B}_{\mathrm{b}}\left(\mathbf{R}_{+}^{k}\right)\right\}$ satisfying $\Lambda([0, t])=X_{t}$ a.s. for $t \in \mathbf{R}_{+}^{k}$. In addition $\Lambda$ is given by

$$
\begin{aligned}
\Lambda(A)= & \int_{A \times \mathbf{R}^{d}} y 1_{D}(y)(J-\operatorname{Leb} \times \nu)(\mathrm{d}(t, y))+\int_{A \times \mathbf{R}^{d}} y 1_{D^{c}}(y) J(\mathrm{~d}(t, y)) \\
& +\int_{A} \mathrm{~d} X_{t}^{\mathrm{g}}+\gamma \operatorname{Leb}(A) \quad \text { a.s. } \quad \text { for } A \in \mathscr{B}_{\mathrm{b}}\left(\mathbf{R}_{+}^{k}\right) .
\end{aligned}
$$

Theorem 2.1,1)-2) are essentially contained in [1, Theorem 4.6]. The only difference is that $J$ above is a Poisson random measure on $\mathbf{R}_{+}^{k} \times \mathbf{R}^{d}$ while Theorem 4.6 of [1] is formulated in terms of Poisson random measures on $\mathbf{R}^{d}$. The proofs are essentially the same and hence we omit the proof of Theorem 2.1,1)-2). See also [5] in the case $k=2$.

For $A \in \mathscr{B}_{\mathrm{b}}\left(\mathbf{R}_{+}^{k}\right)$ define $\int_{A} \mathrm{~d} X_{t}^{\mathrm{g}}=\int 1_{A}(t) \mathrm{d} X_{t}^{\mathrm{g}}$, where we recall that $\int f(t) \mathrm{d} X_{t}^{\mathrm{g}}$ (a random vector in $\mathbf{R}^{d}$ ) is definable by approximation by step functions in the usual way for all measurable $f: \mathbf{R}_{+}^{k} \rightarrow \mathbf{R}$ satisfying $\int(f(t))^{2} \mathrm{~d} t<\infty$. Moreover, we have $\mathscr{L}\left(\int f(t) \mathrm{d} X_{t}^{\mathrm{g}}\right)=N_{d}(0, \Sigma(f))$, where $\Sigma^{i j}(f)=\Sigma^{i j} \int(f(t))^{2} \mathrm{~d} t$. The result in Theorem 2.1,3) is immediate from fundamental properties of integrals with respect to (compensated) Poisson random measures, cf., e.g., [6]. In the case $k=2$, Theorem 2.1,3) can also be found in [5, Theorem 2.6].

We call the process $X^{\mathrm{g}}=\left\{X_{t}^{\mathrm{g}}: t \in \mathbf{R}_{+}^{k}\right\}$ above the Gaussian part of $X$ and the measure $J$ the jump measure of $X$. We also denote it by $J_{X}$. Finally, we call $\Lambda$ above the homogeneous Lévy basis induced by $X$, also to be denoted by $\Lambda_{X}$.

Proposition 2.1. Let $\Lambda=\left\{\Lambda(A): A \in \mathscr{B}_{b}\left(\mathbf{R}_{+}^{k}\right)\right\}$ be a homogeneous Lévy basis. Let $\widetilde{X}_{t}=\Lambda([0, t])$. We then have the following results: For $\left.\left.a \leqslant b, \Delta_{a}^{b} \widetilde{X}=\Lambda(] a, b\right]\right)$ a.s. In particular $\widetilde{X}=\left\{\widetilde{X}_{t}: t \in \mathbf{R}_{+}^{k}\right\}$ is a homogeneous Lévy sheet in law. Let $X=\left\{X_{t}: t \in \mathbf{R}_{+}^{k}\right\}$ be a homogeneous Lévy sheet which is a modification of $\tilde{X}$. Then for $A \in \mathscr{B}_{\mathrm{b}}\left(\mathbf{R}_{+}^{k}\right)$ we have $\Lambda(A)=\Lambda_{X}(A)$ a.s., where $\Lambda_{X}$ is the Lévy basis generated by $X$.

$\mathrm{P}$ r o o f. It is easily seen that $\left.\left.\Delta_{a}^{b} \tilde{X}=\Lambda(] a, b\right]\right)$ a.s. The uniqueness part of Theorem $2.1,3$ ) implies $\Lambda=\Lambda_{X}$.

$\mathrm{R}$ e $\mathrm{m}$ a $\mathrm{rk}$ 2.1. 1) Theorem 2.1,3) shows that a homogeneous Lévy sheet generates a homogeneous Lévy basis by (2.1), and Proposition 2.1 shows conversely that any 
homogeneous Lévy basis is generated in this way. We call (2.1) the Lévy-Itô decomposition of $\Lambda$ and call $J$ in that equation the jump measure of $\Lambda$. We refer to [5] (for the case $k=2$ ) and [7] for the Lévy-Itô decomposition of nonhomogeneous Lévy bases.

2) Let $\Lambda$ be an $\mathbf{R}^{d}$-valued homogeneous Lévy basis on $\mathbf{R}_{+}^{k}$ associated with the characteristic triplet $(\Sigma, \nu, \gamma)$. Assume that $\int_{\mathbf{R}^{d}}(1 \wedge|x|) \nu(\mathrm{d} x)<\infty$. Then for $A \in \mathscr{B}_{\mathrm{b}}\left(\mathbf{R}_{+}^{k}\right)$ the representation (2.1) simplifies to

$$
\Lambda(A)=\int_{A \times \mathbf{R}^{d}} y J(\mathrm{~d}(t, y))+\int_{A} \mathrm{~d} X_{t}^{\mathrm{g}}+\gamma_{0} \operatorname{Leb}(A) \quad \text { a.s. }
$$

where the first integral is defined pointwise almost surely and $\gamma_{0}=\gamma-\int_{A} y 1_{D}(y) \nu(\mathrm{d} y)$. Here pointwise almost surely signifies that, for almost all $\omega, \int_{A \times \mathbf{R}^{d}} y J(\mathrm{~d}(t, y))(\omega)$ is a usual Lebesgue integral. Thus if in addition $\nu\left(\mathbf{R}^{d} \backslash \mathbf{R}_{+}^{d}\right)=0, \Sigma=0$ and $\gamma_{0} \in \mathbf{R}_{+}^{d}$, then we can extend $\Lambda$ in such a way that $\Lambda(A)$ is defined for all $A$ in $\mathscr{B}\left(\mathbf{R}_{+}^{k}\right)$ rather than $\mathscr{B}_{\mathrm{b}}\left(\mathbf{R}_{+}^{k}\right)$; however, some of the coordinates of $\Lambda(A)$ may be equal to $\infty$. In addition, almost surely all coordinates of $\Lambda$ are nonnegative measures.

3. Meta-times. The purpose of this section is to state a result showing that any measure $m$ on $\mathbf{R}_{+}^{k}$ which is finite on compacts is the image measure of Leb under some mapping $\phi$. This result is essentially well known, at least when $m$ is finite, so in the next lemma we simply state a version of it which suits our purposes well.

Lemma 3.1. Let $m=\left\{m(A): A \in \mathscr{B}\left(\mathbf{R}_{+}^{k}\right)\right\}$ be a nonnegative measure on $\mathbf{R}_{+}^{k}$ satisfying $m(\mathscr{A})=0$ and $m(A)<\infty$ for all $A \in \mathscr{B}_{\mathrm{b}}\left(\mathbf{R}_{+}^{k}\right)$. Then there exists a measurable mapping $\phi: \mathbf{R}_{+}^{k} \rightarrow \mathbf{R}^{k}$ such that

$$
m(A)=\operatorname{Leb}\left(\phi^{-1}(A)\right) \quad \text { for all } A \in \mathscr{B}\left(\mathbf{R}_{+}^{k}\right)
$$

and $\phi^{-1}(A)$ is a bounded set for all $A \in \mathscr{B}_{\mathrm{b}}\left(\mathbf{R}_{+}^{k}\right)$.

$\mathrm{R}$ e $\mathrm{m}$ a $\mathrm{r} \mathrm{k}$ 3.1. We refer to the inverse image $\phi^{-1}$ as a meta-time associated with $m$ and we often denote it by $\mathbf{T}$. By the above lemma and properties of inverse images we can regard $\mathbf{T}$ as a mapping $\mathbf{T}: \mathscr{B}_{\mathrm{b}}\left(\mathbf{R}_{+}^{k}\right) \rightarrow \mathscr{B}_{\mathrm{b}}\left(\mathbf{R}_{+}^{k}\right)$ satisfying: (i) $\mathbf{T}(A)$ and $\mathbf{T}(B)$ are disjoint whenever $A, B \in \mathscr{B}_{\mathrm{b}}\left(\mathbf{R}_{+}^{k}\right)$ are disjoint; (ii) $\mathbf{T}\left(\bigcup_{n=1}^{\infty} A_{n}\right)=\bigcup_{n=1}^{\infty} \mathbf{T}\left(A_{n}\right)$ whenever $A_{1}, A_{2}, \ldots$ are in $\mathscr{B}_{\mathrm{b}}\left(\mathbf{R}_{+}^{k}\right)$ and $\bigcup_{n=1}^{\infty} A_{n}$ is in $\mathscr{B}_{\mathrm{b}}\left(\mathbf{R}_{+}^{k}\right)$; (iii) $m(A)=\operatorname{Leb}(\mathbf{T}(A))$ for all $A \in \mathscr{B}_{\mathrm{b}}\left(\mathbf{R}_{+}^{k}\right)$.

P r o o f. Let $u \in \mathbf{R}^{k} \backslash \mathbf{R}_{+}^{k}$ be arbitrary.

1) First assume that $m\left(\mathbf{R}_{+}^{k}\right)<\infty$. Take an interval $[a, b]$ in $\mathbf{R}_{+}^{k}$ with $\operatorname{Leb}([a, b])=$ $m\left(\mathbf{R}_{+}^{k}\right)$. Then $m$ is the image measure of Lebesgue measure on $[a, b]$ under some mapping $\psi:[a, b] \rightarrow \mathbf{R}_{+}^{k}$. That is, $m(A)=\operatorname{Leb}\left(\psi^{-1}(A)\right)$ for all $A \in \mathscr{B}\left(\mathbf{R}_{+}^{k}\right)$. Indeed, this is essentially the well-known result (cf., e.g., [10]) that any distribution on $\mathbf{R}_{+}^{k}$ can be generated from $k$ independent and uniformly distributed random variables. Letting

$$
\phi(t)=\left\{\begin{array}{l}
\psi(t), \quad t \in[a, b] \\
u, \quad t \in \mathbf{R}_{+}^{k} \backslash[a, b],
\end{array}\right.
$$

one sees that $\phi$ has the required properties.

2) If $m\left(\mathbf{R}_{+}^{k}\right)=\infty$ instead, then we can take a sequence $A_{n}, n=1,2, \ldots$, of disjoint bounded Borel sets in $\mathbf{R}_{+}^{k}$ covering $\mathbf{R}_{+}^{k}$ and satisfying that for all $t \in \mathbf{R}_{+}^{k}$ the interval $[0, t]$ is contained in the finite union of some of the $A_{n}$ 's. Define, for all $n \geqslant 1, m_{n}=$ $m\left(\cdot \cap A_{n}\right)$. Since the $m_{n}$ 's are finite measures, there exist a sequence of disjoint intervals $\left[a_{1}, b_{1}\right],\left[a_{2}, b_{2}\right], \ldots$ in $\mathbf{R}_{+}^{k}$ and measurable mappings $\psi_{n}:\left[a_{n}, b_{n}\right] \rightarrow A_{n}$ such that $m_{n}(A)=$ $\operatorname{Leb}\left(\psi_{n}^{-1}(A)\right)$ for all $A \in \mathscr{B}\left(A_{n}\right)$. Since $m=\sum_{n \geqslant 1} m_{n}$, we can define

$$
\phi(t)= \begin{cases}\psi_{n}(t), & t \in\left[a_{n}, b_{n}\right] \text { for some } n, \\ u, & t \in \mathbf{R}_{+}^{k} \backslash\left(\bigcup_{n=1}^{\infty}\left[a_{n}, b_{n}\right]\right) .\end{cases}
$$


Clearly, since for any $t \in \mathbf{R}_{+}^{k}$ the interval $[0, t]$ is contained in the union of a finite number of $A_{n}$ 's it follows that $\phi^{-1}([0, t])$ is contained in the union of a finite number of intervals $\left[a_{n}, b_{n}\right]$.

$\mathrm{E} \times \mathrm{a} \mathrm{p} \mathrm{l}$ e 3.1. Let $m$ be as in the lemma above. In many cases of interest, the mapping $\phi$ in the lemma has a very simple expression, as the following shows.

1) Assume $m$ is concentrated on a set $\mathscr{T}=\left\{t_{n}\right\}_{n=1}^{\infty} \subseteq \mathbf{R}_{+}^{k} \backslash \mathscr{A}$. Take a disjoint sequence $R_{1}, R_{2}, \ldots$ of bounded Borel sets in $\mathbf{R}_{+}^{k}$ such that $\operatorname{Leb}\left(R_{n}\right)=m\left(\left\{t_{n}\right\}\right)$ for all $n$. Define $\phi(t)=t_{n}$ when $t \in R_{n}$ for some $n$ and let $\phi(t)=u$ for $t \in \mathbf{R}_{+}^{k} \backslash\left(\bigcup_{n=1}^{\infty} R_{n}\right)$, where $u \in \mathbf{R}^{k} \backslash \mathbf{R}_{+}^{k}$ is arbitrary. The sets $R_{n}$ can be chosen arbitrarily, showing in particular that $\phi$ is not at all uniquely determined.

2) If $m=$ Leb $/ c$ for some $c>0$, then we can use $\phi(t)=c t$.

3) The case when $m=m_{1}+m_{2}$, where $m_{1}=$ Leb $/ c$ and $m_{2}$ is concentrated on $\left\{t_{n}\right\}_{n=1}^{\infty} \subseteq \mathbf{R}_{+}^{k} \backslash \mathscr{A}$, can be handled as follows. Let the sets $R_{n}$ above be subsets of

$$
\left\{s=\left(s^{1}, \ldots, s^{k}\right) \in \mathbf{R}_{+}^{k}: 0 \leqslant s^{j} \leqslant 1 \text { for all } j=1, \ldots, k\right\} .
$$

Let $e=(1, \ldots, 1) \in \mathbf{R}_{+}^{k}$ be the vector of ones. By defining $\phi$ as

$$
\phi(t)= \begin{cases}t_{n} & \text { if } t \in R_{n} \text { for some } n, \\ u & \text { if } t \in\left\{s=\left(s^{1}, \ldots, s^{k}\right) \in \mathbf{R}_{+}^{k}: s^{j} \in[0,1]\right\} \backslash\left(\bigcup_{n=1}^{\infty} R_{n}\right), \\ c(t-e) & \text { if } t \in\left\{s=\left(s^{1}, \ldots, s^{k}\right) \in \mathbf{R}_{+}^{k}: s^{j}>1\right\},\end{cases}
$$

equation (3.1) is easily verified.

4) Assume $k=1$ and let $T_{t}=m([0, t])$ for all $t \geqslant 0$. Define $\phi: \mathbf{R}_{+} \rightarrow \mathbf{R}$ as

$$
\phi(y)=\inf \{t \geqslant 0: T(t) \geqslant y\},
$$

where inf $\varnothing=u \in \mathbf{R} \backslash \mathbf{R}_{+}$. Then

$$
\left[0, T_{t}\right]=\mathbf{T}([0, t]) \text { for all } t \geqslant 0
$$

and hence $m(A)=\operatorname{Leb}(\mathbf{T}(A))$ for all $A \in \mathscr{B}\left(\mathbf{R}_{+}\right)$.

4. Meta-time changes. In the one-dimensional case $k=1$, one uses increasing functions to model a time change as in (4.3) below. The purpose of the present section is to show that in the case $k \geqslant 2$ certain meta-time changes give similar results. In fact, we show that the appropriate generalization of the process $Y$ in (4.3) is the random measure $M$ in (4.1), where in the latter equation $\mathbf{T}$ is a meta-time as defined in Section 3.

Let $X=\left\{X_{t}: t \in \mathbf{R}_{+}^{k}\right\}$ be an $\mathbf{R}^{d}$-valued homogeneous Lévy sheet on $\mathbf{R}_{+}^{k}$ associated with $\mu \in \operatorname{ID}\left(\mathbf{R}^{d}\right)$. Denote the corresponding homogeneous Lévy basis by $\Lambda_{X}$. Let $m=$ $\left\{m(A): A \in \mathscr{B}\left(\mathbf{R}_{+}^{k}\right)\right\}$ be a nonnegative measure on $\mathbf{R}_{+}^{k}$ satisfying $m(\mathscr{A})=0$ and $m(A)<$ $\infty$ for all $A \in \mathscr{B}_{\mathrm{b}}\left(\mathbf{R}_{+}^{k}\right)$. Set $T_{t}=m([0, t])$ for all $t \in \mathbf{R}_{+}^{k}$ and let $\phi: \mathbf{R}_{+}^{k} \rightarrow \mathbf{R}^{k}$ be given as in Lemma 3.1. Let $\mathbf{T}=\phi^{-1}$ be the corresponding meta-time associated with $m$.

Define $M=\left\{M(A): A \in \mathscr{B}_{b}\left(\mathbf{R}_{+}^{k}\right)\right\}$ as

$$
M(A)=\Lambda_{X}(\mathbf{T}(A)) \quad \text { for } A \in \mathscr{B}_{\mathrm{b}}\left(\mathbf{R}_{+}^{k}\right) .
$$

Using the properties of $\mathbf{T}$ in Remark 3.1 one sees that $M$ is a (nonhomogeneous) Lévy basis. Since in addition $\Lambda_{X}$ is a homogeneous Lévy basis associated with $\mu$, it follows that

$$
\mathscr{L}(M(A))=\mu^{m(A)} \quad \text { for } A \in \mathscr{B}_{\mathrm{b}}\left(\mathbf{R}_{+}^{k}\right) .
$$

In particular, for $t \in \mathbf{R}_{+}^{k}, M((0, t])$ has characteristic triplet $T_{t}(\Sigma, \nu, \gamma)$, where $(\Sigma, \nu, \gamma)$ is the characteristic triplet of $\mu$. We say that $M$ is defined from $\Lambda_{X}$ by meta-time change with $\mathbf{T}$.

$\mathrm{R}$ e $\mathrm{m}$ a $\mathrm{r} \mathrm{k}$ 4.1. Let $k=1$ and let us show that in this case the above definition generalizes the usual concept of a time change in a natural way. For this purpose, define a process $Y=\left\{Y_{t}: t \geqslant 0\right\}$ by time changing $X$ with $T$ :

$$
Y_{t}=X_{T_{t}} \text {. }
$$


Hence, $Y$ is a càdlàg process with independent increments and it is an additive process (i.e., also continuous in probability) if $T$ is continuous. The distribution of an increment is given as

$$
\mathscr{L}\left(Y_{t}-Y_{s}\right)=\mu^{T_{t}-T_{s}} \quad \text { for } 0 \leqslant s<t .
$$

Let $\phi$ be given as in Example 3.1,4). Using (3.2) rewrite $Y_{t}$ in terms of the Lévy basis $\Lambda_{X}$ as

$$
Y_{t}=\Lambda_{X}\left(\left[0, T_{t}\right]\right)=\Lambda_{X}(\mathbf{T}([0, t])) \quad \text { for } t \geqslant 0 .
$$

This shows that (4.1) provides a natural generalization of (4.3) and (4.5), since we simply replace $[0, t]$ by an arbitrary bounded Borel set; in return we get a measure $M$ instead of a process $Y$. Similarly, (4.4) is generalized by (4.2).

$\mathrm{R}$ e $\mathrm{m}$ a $\mathrm{rk}$ 4.2. There are many alternative representations of $M$ and in the following we consider some of them. Let $\left\{L_{t}: t \geqslant 0\right\}$ denote an $\mathbf{R}^{d}$-valued Lévy process with $\mu=\mathscr{L}\left(L_{1}\right)$. Thus, in the language of [2], $\left\{L_{t}: t \geqslant 0\right\}$ is a Lévy seed associated with $\mu$.

1) If $A_{1}, \ldots, A_{r}$ are disjoint bounded Borel sets, then

$$
\left(M\left(A_{1}\right), \ldots, M\left(A_{r}\right)\right) \stackrel{\mathscr{D}}{=}\left(L_{m\left(A_{1}\right)}^{A_{1}}, \ldots, L_{m\left(A_{r}\right)}^{A_{r}}\right)
$$

where $\left\{L_{t}^{A_{j}}: t \geqslant 0\right\}$, for $j=1, \ldots, r$, are independent copies of $\left\{L_{t}: t \geqslant 0\right\}$. This follows since $\mathscr{L}\left(L_{m\left(A_{j}\right)}^{A_{j}}\right)=\mu^{m\left(A_{j}\right)}$. If $A_{1} \subseteq A_{2} \subseteq \cdots \subseteq A_{r}$ instead, then

$$
\left(M\left(A_{1}\right), \ldots, M\left(A_{r}\right)\right) \stackrel{\mathscr{D}}{=}\left(L_{m\left(A_{1}\right)}, \ldots, L_{m\left(A_{r}\right)}\right) .
$$

2) Consider the case where $m$ is given as in Example 3.11); that is, $m$ is concentrated on $\mathscr{T}=\left\{t_{n}\right\}_{n=1}^{\infty} \subseteq \mathbf{R}_{+}^{k} \backslash \mathscr{A}$. For $A \in \mathscr{B}_{b}\left(\mathbf{R}_{+}^{k}\right)$ we then have

$$
\begin{aligned}
M(A) & =\Lambda_{X}\left(\mathbf{T}\left(A \cap \mathscr{T}^{c}\right)\right)+\Lambda_{X}(\mathbf{T}(A \cap \mathscr{T})) \\
& =\Lambda_{X}(\mathbf{T}(A \cap \mathscr{T}))=\sum_{n: t_{n} \in A} \Lambda_{X}\left(\mathbf{T}\left(\left\{t_{n}\right\}\right)\right) \quad \text { a.s. }
\end{aligned}
$$

where the series converges almost surely and the first term on the right-hand side of (4.6) vanishes, since $\operatorname{Leb}\left(\mathbf{T}\left(A \cap \mathscr{T}^{c}\right)\right)=0$ by (3.1). Since, by the same equation, $\operatorname{Leb}\left(\mathbf{T}\left(\left\{t_{n}\right\}\right)\right)=$ $m\left(\left\{t_{n}\right\}\right)$, we have $\Lambda_{X}\left(\mathbf{T}\left(\left\{t_{n}\right\}\right)\right) \stackrel{\mathscr{D}}{=} L_{m\left(\left\{t_{n}\right\}\right)}$. Taking a sequence $\left\{L_{t}^{n}: t \geqslant 0\right\}, n=1,2, \ldots$, of independent copies of $\left\{L_{t}: t \geqslant 0\right\}$ we thus have for all $r \geqslant 1$ and $A_{1}, \ldots, A_{r} \in \mathscr{B}_{\mathrm{b}}\left(\mathbf{R}_{+}^{k}\right)$ that

$$
\left(M\left(A_{1}\right), \ldots, M\left(A_{r}\right)\right) \stackrel{\mathscr{D}}{=}\left(\sum_{n: t_{n} \in A_{1}} L_{m\left(\left\{t_{n}\right\}\right)}^{n}, \ldots, \sum_{n: t_{n} \in A_{r}} L_{m\left(\left\{t_{n}\right\}\right)}^{n}\right) .
$$

If $\mu=N_{d}(\gamma, I)$ (where $I$ is the $d \times d$ identity matrix), then this simplifies as follows. Let $\varepsilon_{1}, \varepsilon_{2}, \ldots$ denote a sequence of independent and identically distributed random vectors with law $N_{d}(0, I)$. Then (4.7) is equivalent to

$$
\begin{aligned}
& \left(M\left(A_{1}\right), \ldots, M\left(A_{r}\right)\right) \\
& \quad \stackrel{\mathscr{D}}{=}\left(\sum_{n: t_{n} \in A_{1}} \gamma m\left(\left\{t_{n}\right\}\right)+\left[m\left(\left\{t_{n}\right\}\right)\right]^{1 / 2} \varepsilon_{n}, \ldots, \sum_{n: t_{n} \in A_{r}} \gamma m\left(\left\{t_{n}\right\}\right)+\left[m\left(\left\{t_{n}\right\}\right)\right]^{1 / 2} \varepsilon_{n}\right) .
\end{aligned}
$$

3) Finally consider the case $m=m_{1}+m_{2}$ as in Example 1,3), where $m_{1}=$ Leb $/ c$ and $m_{2}$ is concentrated on $\mathscr{T}$. Then $M=M_{1}+M_{2}$, where $M_{i}=\left\{M_{i}(A): A \in \mathscr{B}_{b}\left(\mathbf{R}_{+}^{k}\right)\right\}$ for $i=$ 1,2 are independent and given by $M_{1}(A)=\Lambda_{X}\left(\mathbf{T}\left(A \cap \mathscr{T}^{c}\right)\right)$ and $M_{2}(A)=\Lambda_{X}(\mathbf{T}(A \cap \mathscr{T}))$ for $A \in \mathscr{B}_{b}\left(\mathbf{R}_{+}^{k}\right)$. The measure $M_{1}$ is a homogeneous Lévy basis associated with $\mu^{1 / c}$ and $M_{2}$ can be represented as $M$ in 2).

$\mathrm{R}$ e $\mathrm{m}$ a r k 4.3. From the Lévy-Itô decomposition (2.1) of $\Lambda_{X}$ we have, a.s. for $A \in \mathscr{B}_{\mathrm{b}}\left(\mathbf{R}_{+}^{k}\right)$,

$$
M(A)=\int_{\mathbf{T}(A) \times \mathbf{R}^{d}} y 1_{D}(y)\left(J_{X}-\operatorname{Leb} \times \nu\right)(\mathrm{d}(t, y))+\int_{\mathbf{T}(A) \times \mathbf{R}^{d}} y 1_{D^{c}}(y) J_{X}(\mathrm{~d}(t, y))
$$




$$
+\int_{\mathbf{T}(A)} \mathrm{d} X_{t}^{\mathrm{g}}+\gamma \operatorname{Leb}(\mathbf{T}(A))
$$

Applying the transformation rule on the first two integrals we get the following Lévy-Itô type representation of $M$ :

$$
\begin{aligned}
M(A)= & \int_{A \times \mathbf{R}^{d}} y 1_{D}(y)\left(\widetilde{J}_{X}-m \times \nu\right)(\mathrm{d}(t, y))+\int_{A \times \mathbf{R}^{d}} y 1_{D^{c}}(y) \widetilde{J}_{X}(\mathrm{~d}(t, y)) \\
& +\int_{\mathbf{T}(A)} \mathrm{d} X_{t}^{\mathrm{g}}+\gamma m(A) \quad \text { a.s. } \quad \text { for } A \in \mathscr{B}_{\mathrm{b}}\left(\mathbf{R}_{+}^{k}\right)
\end{aligned}
$$

where $\widetilde{J}_{X}=\left\{\widetilde{J}_{X}(C): C \in \mathscr{B}\left(\mathbf{R}_{+}^{k} \times \mathbf{R}^{d}\right)\right\}$ is the Poisson random measure given by $\widetilde{J}_{X}(A \times$ $B)=J_{X}(\mathbf{T}(A) \times B)$ for all $A \in \mathscr{B}_{\mathrm{b}}\left(\mathbf{R}_{+}^{k}\right)$ and $B \in \mathscr{B}_{\mathrm{b}}\left(\mathbf{R}^{d}\right)$.

5. Extended subordination. Let $X=\left\{X_{t}: t \in \mathbf{R}_{+}^{k}\right\}, \Lambda_{X}=\left\{\Lambda_{X}(A): A \in\right.$ $\left.\mathscr{B}_{b}\left(\mathbf{R}_{+}^{k}\right)\right\}, \mu$ and $(\Sigma, \nu, \gamma)$ be given as in the previous section. That is, $X$ is an $\mathbf{R}^{d}$ valued homogeneous Lévy sheet on $\mathbf{R}_{+}^{k}$ associated with $\mu$, which has characteristic triplet $(\Sigma, \nu, \gamma)$, and $\Lambda_{X}$ is the homogeneous Lévy basis induced by $X$. Let $T=\left\{T_{t}: t \in \mathbf{R}_{+}^{k}\right\}$ be an $\mathbf{R}_{+}$-valued homogeneous Lévy sheet associated with a distribution $\lambda \in \operatorname{ID}(\mathbf{R})$. Let $\lambda$ have Lévy measure $\rho$ and drift $\beta \in \mathbf{R}_{+}$; that is, $\rho\left(\mathbf{R}_{-}\right)=0, \int_{\mathbf{R}_{+}}(1 \wedge x) \rho(\mathrm{d} x)<\infty$ and

$$
\widehat{\lambda}(u)=\exp \left[\mathrm{i} \beta u+\int_{\mathbf{R}_{+}}\left(e^{\mathrm{i} u x}-1\right) \rho(\mathrm{d} x)\right] \text { for } u \in \mathbf{R} .
$$

Let $\Lambda_{T}=\left\{\Lambda_{T}(A): A \in \mathscr{B}\left(\mathbf{R}_{+}^{k}\right)\right\}$ be the nonnegative homogeneous Lévy basis induced by $T=\left\{T_{t}: t \in \mathbf{R}_{+}^{k}\right\}$. By removing a null set if necessary it follows from Remark 2.12) that $\Lambda_{T}$ has the pointwise representation

$$
\begin{aligned}
\Lambda_{T}(A)(\omega) & =\int_{A \times \mathbf{R}_{+}} y J_{T}(\mathrm{~d}(t, y))(\omega)+\beta \operatorname{Leb}_{1}(A) \\
& =\sum_{t \in A} \Lambda_{T}(\{t\})(\omega)+\beta \operatorname{Leb}_{1}(A) \quad \text { for } \omega \in \Omega \text { and } A \in \mathscr{B}\left(\mathbf{R}_{+}^{k}\right)
\end{aligned}
$$

where Leb 1 denotes Lebesgue measure on $\mathbf{R}_{+}$and the series converges for all $A \in \mathscr{B}_{\mathrm{b}}\left(\mathbf{R}_{+}^{k}\right)$ and $\omega \in \Omega$. Let $\mathscr{F}^{T}=\sigma\left(\Lambda_{T}(A): A \in \mathscr{B}_{\mathrm{b}}\left(\mathbf{R}_{+}^{k}\right)\right)$ be the sigma-field generated by $\Lambda_{T}$.

Pointwise the measure $A \rightarrow \Lambda_{T}(A)(\omega)$ is the sum of a discrete measure and a constant times Lebesgue measure. By the construction in Example 3.1,3) there is an $\left(\mathscr{F}^{T} \times\right.$ $\mathscr{B}\left(\mathbf{R}_{+}^{k}\right), \mathscr{B}\left(\mathbf{R}^{k}\right)$ )-measurable mapping $\phi_{T}: \Omega \times \mathbf{R}_{+}^{k} \rightarrow \mathbf{R}^{k}$ such that for all $\omega \in \Omega$ and $A \in \mathscr{B}_{\mathrm{b}}\left(\mathbf{R}_{+}^{k}\right)$ the set $\mathbf{T}(A)(\omega)$, given by $\mathbf{T}(A)(\omega)=\left\{x \in \mathbf{R}_{+}^{k}: \phi_{T}(\omega, x) \in A\right\}$, is bounded, and

$$
\Lambda_{T}(A)(\omega)=\operatorname{Leb}(\mathbf{T}(A)(\omega)) .
$$

That is, for each $\omega, \mathbf{T}(\cdot)(\omega)$ is a meta-time associated with $\Lambda_{T}(\cdot)(\omega)$.

Define $M=\left\{M(A): A \in \mathscr{B}_{\mathrm{b}}\left(\mathbf{R}_{+}^{k}\right)\right\}$ as

$$
M(A)=\Lambda_{X}(\mathbf{T}(A)) \quad \text { for } A \in \mathscr{B}_{\mathrm{b}}\left(\mathbf{R}_{+}^{k}\right),
$$

where as usual we suppress $\omega$ on both sides. We say that $M$ appears by extended subordination of $\Lambda_{X}$ by $\Lambda_{T}$ or of $X$ by $T$; and we write $M=\Lambda_{X} \curlywedge \Lambda_{T}$ or $M=X \curlywedge T$.

In practice the meta-time $\mathbf{T}$ can be hard to work with directly. Therefore it is important to note that if we condition on $T$, then, by (5.1), the useful representations of $M$ in Remark 4. apply. For example, if $\lambda$ above is a Poisson or negative binomial distribution, then almost surely $\Lambda_{T}$ is concentrated on a finite number of points on compacts. If $\lambda$ is a gamma or an inverse Gaussian distribution, then almost surely $\Lambda_{T}$ is concentrated on a dense subset of $\mathbf{R}_{+}^{k}$.

The following corresponds to the theorem in [2, Section 3.1]. 
Theorem 5.1. Assume $M=\Lambda_{X} \curlywedge \Lambda_{T}$ as above. Then $M=\left\{M(A): A \in \mathscr{B}_{\mathrm{b}}\left(\mathbf{R}_{+}^{k}\right)\right\}$ is a homogeneous Lévy basis associated with the measure $\mu^{\#} \in \operatorname{ID}\left(\mathbf{R}^{d}\right)$ with characteristic triplet $\left(\Sigma^{\#}, \nu^{\#}, \gamma^{\#}\right)$, where

$$
\begin{aligned}
\Sigma^{\#}=\beta \Sigma, \quad \nu^{\#}(B) & =\beta \nu(B)+\int_{0}^{\infty} \mu^{s}(B) \rho(\mathrm{d} s), \quad B \in \mathscr{B}\left(\mathbf{R}^{d} \backslash\{0\}\right), \\
\gamma^{\#} & =\beta \gamma+\int_{0}^{\infty} \int_{|x| \leqslant 1} x \mu^{s}(\mathrm{~d} x) \rho(\mathrm{d} s)
\end{aligned}
$$

P r o o f. Conditionally on $\mathscr{F}^{T}$, and hence also unconditionally, $M$ satisfies the $\sigma$ additivity condition in Definition 2.2 (ii).

Let $n \geqslant 1$, and let $A_{1}, \ldots, A_{n} \in \mathscr{B}_{\mathrm{b}}\left(\mathbf{R}_{+}^{k}\right)$ be disjoint. Conditionally on $\mathscr{F}^{T}$ we are in the setting of the previous section. That is, $M\left(A_{1}\right), \ldots, M\left(A_{n}\right)$ are independent conditionally on $\mathscr{F}^{T}$ and $\mathscr{L}\left(M\left(A_{j}\right) \mid \mathscr{F}^{T}\right)=\mu^{\Lambda_{T}\left(A_{j}\right)}$. Therefore, for arbitrary $z_{1}, \ldots, z_{n} \in$ $\mathbf{R}^{d}$ we have

$$
\mathbf{E}\left[\prod_{j=1}^{n} e^{\mathrm{i}\left\langle z_{j}, M\left(A_{j}\right)\right\rangle} \mid \mathscr{F}^{T}\right]=\prod_{j=1}^{n} \widehat{\mu}\left(z_{j}\right)^{\Lambda_{T}\left(A_{j}\right)} .
$$

Since $\Lambda_{T}\left(A_{1}\right), \ldots, \Lambda_{T}\left(A_{n}\right)$ are independent, it thus follows that

$$
\mathbf{E}\left[\prod_{j=1}^{n} e^{\mathrm{i}\left\langle z_{j}, M\left(A_{j}\right)\right\rangle}\right]=\prod_{j=1}^{n} \mathbf{E}\left[\widehat{\mu}\left(z_{j}\right)^{\Lambda_{T}\left(A_{j}\right)}\right],
$$

showing that $M\left(A_{1}\right), \ldots, M\left(A_{n}\right)$ are independent. Since, moreover, $\mathscr{L}\left(\Lambda_{T}(A)\right)=$ $\mathscr{L}\left(\Lambda_{T}(t+A)\right)$ for all $t \in \mathbf{R}_{+}^{k}$ and $A \in \mathscr{B}_{\mathrm{b}}\left(\mathbf{R}_{+}^{k}\right)$, it follows that $\mathscr{L}(M(A))=\mathscr{L}(M(t+A))$. Thus, $M$ is a homogeneous Lévy basis.

Choose an arbitrary set $A \in \mathscr{B}_{\mathrm{b}}\left(\mathbf{R}_{+}^{k}\right)$ with $\operatorname{Leb}(A)=1$. Then $\mu^{\#}=\mathscr{L}(M(A))$ and by the above we have for $z \in \mathbf{R}^{d}$ that $\widehat{\mu^{\#}}(z)=\mathbf{E}\left[\widehat{\mu}(z)^{\Lambda_{T}(A)}\right]$.

Let $\left\{L_{t}: t \geqslant 0\right\}$ be a Lévy process with $\mathscr{L}\left(L_{1}\right)=\mu$ and let $\left\{H_{t}: t \geqslant 0\right\}$ be a subordinator independent of $L$ with $\mathscr{L}\left(H_{1}\right)=\lambda$. It is easily seen that $\mathbf{E}\left[e^{\mathrm{i}\left\langle z, L_{H_{1}}\right\rangle}\right]=\mathbf{E}\left[\widehat{\mu}(z)^{H_{1}}\right]=$ $\widehat{\mu^{\#}}(z)$. In other words $\mathscr{L}\left(L_{H_{1}}\right)=\mu^{\#}$, which means that $\mu^{\#}$ appears as the law of a subordinated process in the usual sense. It is therefore well known, e.g., from [11, Theorem 30.1], that the characteristic triplet of $\mu^{\#}$ is as indicated.

$\mathrm{R}$ e $\mathrm{m}$ a r k 5.1. Above we assumed that $\Lambda_{T}$ is a nonnegative homogeneous Lévy basis; however, it is possible to define $M=\Lambda_{X} \curlywedge \Lambda_{T}$ in a much more general context. For example, assume that $\Lambda_{T}=\left\{\Lambda_{T}(A): A \in \mathscr{B}_{\mathrm{b}}\left(\mathbf{R}_{+}^{k}\right)\right\}$ is stationary in the sense that $\left\{\Lambda_{T}(A): A \in \mathscr{B}_{\mathrm{b}}\left(\mathbf{R}_{+}^{k}\right)\right\} \stackrel{\mathscr{D}}{=}\left\{\Lambda_{T}(t+A): A \in \mathscr{B}_{\mathrm{b}}\left(\mathbf{R}_{+}^{k}\right)\right\}$ for all $t \in \mathbf{R}_{+}^{k}$ and that for all $\omega$ $A \mapsto \Lambda_{T}(A)(\omega)$ is a nonnegative measure on $\mathbf{R}_{+}^{k}$. Assume $\Lambda_{T}$ and $\Lambda_{X}$ are independent and note that we do no longer assume that $\Lambda_{T}$ is a Lévy basis. Using Lemma 3.1 define $\phi_{T}: \Omega \times \mathbf{R}_{+}^{k} \rightarrow \mathbf{R}^{k}$ such that we have (5.2) and let $M=\left\{M(A): A \in \mathscr{B}_{\mathrm{b}}\left(\mathbf{R}_{+}^{k}\right)\right\}$ be given as in (5.3). Then $M=\left\{M(A): A \in \mathscr{B}_{\mathrm{b}}\left(\mathbf{R}_{+}^{k}\right)\right\}$ is a homogeneous random measure in the sense that for any disjoint sequence $A_{1}, A_{2}, \ldots$ with $A=\bigcup_{n=1}^{\infty} A_{n} \in \mathscr{B}_{\mathrm{b}}\left(\mathbf{R}_{+}^{k}\right)$ we have $M(A)=\sum_{n=1}^{\infty} M\left(A_{n}\right)$ a.s. Moreover, by a slight modification of the above proof it follows that $M$ is stationary. In general $M$ is no longer a Lévy basis. But conditionally on $T$ it is.

\section{REFERENCES}

1. Adler R.J., Monrad D., Scissors R.H., Wilson R. Representations, decompositions and sample function continuity of random fields with independent increments. Stochastic Process. Appl., 1983, v. 15, № 1, pp. 3-30.

2. Barndorff-Nielsen O.E. Lévy Bases and Extended Subordination. Research Report № 12. Aarhus: Thiele Centre for Applied Mathematics in Natural Science, 2010, 15 p. 
3. Barndorff-Nielsen O.E., Pedersen J., Sato K. Multivariate subordination, selfdecomposability and stability. - Adv. Appl. Probab., 2001, v. 33, № 1, pp. 160-187.

4. Barndorff-Nielsen O. E., Shiryaev A.N. Change of Time and Change of Measure. Hackensack: World Scientific, 2010, 305 p.

5. Dalang R. C., Walsh J. B. The sharp Markov property of Lévy sheets. - Ann. Probab. 1992 , v. 20 , № 2 , p. 591-626.

6. Jacod J., Shiryaev A.N. Limit Theorems for Stochastic Processes. Berlin: SpringerVerlag, 2003, 661 p. (Grundlehren Math. Wiss., v. 288.)

7. Pedersen $J$. The Lévy-Itô decomposition of an independently scattered random measure. - Research Report № 2. Aarhus: MaPhySto, 2003.

8. Pedersen J., Sato K. Relations between cone-parameter Lévy processes and convolution semigroups. - J. Math. Soc. Japan, 2004, v. 54, № 2, p. 541-559.

9. Rajput B.S., Rosinski J. Spectral representations of infinitely divisible processes. Probab. Theory Related Fields, 1989, v. 82, № 3, p. 451-487.

10. Rosenblatt $M$. Remarks on a multivariate transformation. - Ann. Math. Statist., 1952 , v. 23 , p. $470-472$.

11. Sato K. Lévy Processes and Infinitely Divisible Distributions. Cambridge: Cambridge Univ. Press, 1999, 486 p. (Cambridge Stud. Adv. Math., v. 68.)

Поступила в редакцию 24.XII. 2010

(c) $2011 \Gamma$

ZHANG Z.*

\title{
BOUNDS FOR CHARACTERISTIC FUNCTIONS AND LAPLACE TRANSFORMS OF PROBABILITY DISTRIBUTIONS
}

\begin{abstract}
Используя обобщение неравенства типа Рао-Крамера, мы устанавливаем некоторые неравенства, связывающие характеристические функции и преобразования Лапласа решетчатых распределений. Мы также указываем, как эти результаты могут быть распространены на случай непрерывных плотностей, и иллюстрируем некоторые применения.
\end{abstract}

Ключевые слова и фразы: характеристическая функция, преобразование Лапласа, решетчатое распределение, информация Фишера, неравенство.

1. Introduction. The apparatus of characteristic functions is of fundamental importance in probability theory, it provides a powerful analytical tool in analyzing random phenomena. In this paper, we will focus on establishing several inequalities for comparing characteristic functions and Laplace transforms of probability distributions.

Let $F$ be a probability distribution function, that is, $F: \mathbf{R} \rightarrow[0,1]$ is nondecreasing, right continuous, with $\lim _{t \rightarrow-\infty} F(t)=0$ and $\lim _{t \rightarrow \infty} F(t)=1$. Let

$$
\phi(t)=\int_{\mathbf{R}} e^{i t x} d F(x), \quad t \in \mathbf{R},
$$

be its characteristic function [2], [7] and put

$$
\psi(r)=\int_{\mathbf{R}} e^{-r x} d F(x), \quad r \in \mathbf{R},
$$

if the integral is finite. When $F$ is supported on the positive (negative) real axis, $\psi(r)$ is always well defined for $r \geqslant 0(r \leqslant 0)$, and in this case, $\psi$ is sometimes referred to as the generating function of $F$.

* School of Mathematics and Statistics, Carleton University, Ottawa, ON, K1S 5B6, canada; e-mail: zhangzm@math.carleton.ca 